\title{
$\checkmark$ Research Square \\ Safety and Efficacy of 25G vitrectomy surgery for malignant glaucoma in pseudophakia
}

fei you(ロ379620200@qq.com )

Research article

Keywords: cataract extraction, malignant glaucoma, vitrecomy

Posted Date: August 23rd, 2019

DOI: https://doi.org/10.21203/rs.2.13438/v1

License: (9) (i) This work is licensed under a Creative Commons Attribution 4.0 International License.

Read Full License 


\section{Abstract}

Background: malignant glaucoma after cataract surgery is still one of the serious complications, if not handled properly 囚it may lead to serious consequences. It is notoriously difficult to treat. 25G vitrectomy was performed to evaluate the safety and efficacy for the treatment of malignant glaucoma in pseudophakia.

Methods: This is a retrospective, comparative case series study. A total of 20 eyes of 20 patients with malignant glaucoma after phacoemulsification were analyzed retrospectively in The First Affiliated Hospital Of Anhui Medical University from May 2015 to January 2018. All Medical Data including the best corrected visual acuity (BCVA), Change of intraocular pressure (IOP), the length of eye axis, and the depth of anterior chamber were recorded. SPSS 17.0 statistical software was used for analysis .Before surgery, the best corrected visual acuity (BCVA) was 1.8 \pm 0.6 . The intraocular pressure was between 18$57 \mathrm{mmHg}$, with an average of $35.2 \pm 10.4 \mathrm{mmHg}$.The depth of anterior chamber was between $0.9-1.9 \mathrm{~mm}$, with an average of $1.3 \pm 0.2 \mathrm{~mm}$. The length of eye axis was $19.7-22.5 \mathrm{~mm}$,with an average of $20.6 \pm 0.5 \mathrm{~mm}$. All the patients were accomplished with $25 \mathrm{G}$ vitrectomy. Besides, anterior chamber inflammatory reaction and other complications were also observed postoperation.

Results: The patients were followed up for 6-12 months with an average of 9 months. BCVA at the last follow up improved to $0.8 \pm 0.1$, and there was significant difference compared to that before operation $(P<0.01)$.IOP was from $12-19 \mathrm{mmHg}$, an average of $16.1 \pm 2.5 \mathrm{mmHg}$, there was significant difference compared to that before operation $(t=7.6, p<0.01)$. Only one patient occurred low IOP $(6 \mathrm{mmHg})$ after surgery, IOP returned to normal level $(14 \mathrm{mmHg})$ after conservative treatment. No serious complications including corneal endothelium decompensation, intraocular lens (IOL) capture, intraocular hemorrhage, endophthalmitis and uncontrolled IOP were observed.

Conclusions: $25 \mathrm{G}$ minimally invasive vitrectomy can treat malignant glaucoma after cataract surgery safely and effectively

\section{Backgound}

Cataract is the first cause of blindness in the word. Surgical treatment is the only effective way to treat cataract. With the development of science and technology, there are fewer and fewer complications caused by cataract surgery. However, malignant glaucoma after cataract surgery is still one of the serious complications, if not handled properly ¿it may lead to serious consequences. It is notoriously difficult to treat ${ }^{[1]}$. In recent years, $25 \mathrm{G}$ minimally invasive vitrectomy has been used to treat malignant glaucoma after cataract surgery, and good results have been achieved. The results are as follows.

\section{Methods}

\section{1 Clinical data}


A total of 20 eyes of 20 patients with malignant glaucoma after phacoemulsification in The First Affiliated Hospital Of Anhui Medical University from May 2015 to January 2018 were recruited, aged 5078 , with a average of $64 \pm 0.4$. The study was approved by the Institutional Review Board and Ethics committee of Anhui Medical University. There were 12 males and 8 females. Among them, 9 cases were right eye and 11 cases were left eye. trabeculectomy had been performed in 4 out of 20 eyes. The best corrected visual acuity (BCVA) was $1.8 \pm 0.6$. The intraocular pressure was between $18-57 \mathrm{mmHg}$, with an average of $35.2 \pm 10.4 \mathrm{mmHg}$. The depth of anterior chamber was between $0.9-1.9 \mathrm{~mm}$, with an average of $1.3 \pm 0.2 \mathrm{~mm}$. Postoperative malignant glaucoma occurred 4 days -3 months after phacoemulsification combined with intraocular lens implantation, with a median time of 9 days.

Diagnostic criteria: (1) After cataract surgery, central and peripheral anterior chambers became consistently shallow, even the anterior chamber disappeared completely, irido-cilio-vitreal diaphapharagm moved forward, with normal or elevated intraocular pressure; (2) The treatment of miotic was invalid or aggravated the condition. The treatment of hyperosmolar can decrease the intraocular pressure in a short time. The treatment of cycloplegia can relieve the symptoms of the patients; malignant glaucoma can be diagnosed if the patient had both of the above-mentioned characteristics and pupil block, suprachoroidal leakage or bleeding, and so on were excluded.

\section{- Preoperative management}

Cycloplegia was prescribed to move the irido-cilio-vitreal diaphapharagm backwards and widen the middle segment of the eye. Methazolamide Tablets $25 \mathrm{mg}$ and Catilol hydrochloride eye drops were adopted twice a day. Intravenous injection of $20 \%$ solution of Mannitol injection $250 \mathrm{ml}$ was administered once a day. Laser posterior capsulotomy was performed in patients who had not been remitted for 3-7 days. Only 1 eye of 20 patients had stable intraocular pressure after conservative treatment, but relapsed after withdrawal. The other 19 eyes were treated with $25 \mathrm{G}$ minimally invasive vitrectomy. The patient could not stop using atropine and anti-glaucoma medications after conservative treatment for 1 month and was treated with surgery.

\subsection{Surgical method}

All patients were operated by the same experienced Ophthalmologist. The posterior capsule was incised with a $25 \mathrm{G}$ vitrectomy knife. The anterior chamber was deepened. The incision diameter standard is whether the anterior chamber deepening can be observed as a standard. The anterior vitreous boundary membrane and anterior vitreous body were removed. With iris root incision, the vitreous body around the root incision should be excised to keep the root incision unobstructed. The cannulas were pulled out after observing no abnormalities in the retina. All the punctures were not sutured. After the operation, glucocorticoid, nonsteroidal anti-inflammatory drugs and compound tropiamine eye drops were used.

\subsection{Observation indicators}


The patients were followed up for 6-12 months with an average of 9 months. Visual acuity, IOP, the length of eye axis, the depth of anterior chamber, complications were observed pre- and postoperation.

\section{Statistical analysis}

SPSS 17.0 statistical software was used for analysis. BCVA was recorded as decimal values and converted to logarithm of the minimum angle of resolution (log MAR) for statistical anaylsis. The measurement data were expressed by mean \pm standard deviation, and paired sample $T$ test were adopted. With $P<0.05$ as the difference was statistically significant.

\section{Results}

\section{- Visual acuity}

The BCVA was $1.8 \pm 0.6$ preoperation. During the last follow up, the BCVA improved to $0.8 \pm 0.1$, the difference was statistically significant $(t=8.3, p<0.05)$.

\section{- anterior chamber depth}

At the last follow-up, the depth of the anterior chamber was $2.4 \pm 0.5 \mathrm{~mm}$, and the depth of the anterior chamber was $1.3 \pm 0.2 \mathrm{~mm}$ preoperation. The depth of the anterior Chamber of the patients after surgery was significantly deepened, and the difference was statistically significant $(t=9.1, p<0.01)$.

\subsection{Intraocular pressure}

At the last follow-up, the IOP was $16.1 \pm 2.5$, and the preoperative Intraocular pressure was $35.2 \pm 10.4$. The IOP after surgery was significantly reduced, and the difference was statistically significant $(t=7.6, p<0.01)$

\subsection{Complications}

In all patients, there was mild inflammatory reaction in the anterior chamber one day postoperation. After drip with non-steroidal anti-inflammatory drugs and glucocorticoids, Symptoms were alleviated. the IOP in one patient was low, which was $6 \mathrm{mmHg}$. After local and systemic use of glucocorticoid, it returned to normal. After 3 days, the IOP was $15 \mathrm{mmmHg}$.

\section{Discussion}

Malignant glaucoma, also known as ciliary cycloobstructive glaucoma, was first described by VonGraefe ${ }^{[2]}$ and is a rare but very serious glaucoma. The most common occurrence is after anti-glaucoma filtration surgery ${ }^{[3]}$. It can also be observed after cataract phacoemulsification combined with intraocular lens implantation, eye trauma, and the use of miotic ${ }^{[4-6]}$. To sum up, there were total of about 12000 cataract phacoemulsification combined with intraocular lens implantation from 2015 to 2018,20 cases of 
malignant glaucoma occurred, the incidence rate was $0.016 \%$, and the occurrence time was 4 days to 3 months postoperation. The results of the study were basically consistent with the relevant reports ${ }^{[7]}$.

After malignant glaucoma occurs, it is generally manifested as a shallowness of the anterior chamber, elevated or normal ocular pressure [8]. The application of miotic can not reduce IOP, but leads to further increase of ocular pressure. The pathogenesis of malignant glaucoma is complex, and the etiology is still unclear ${ }^{[8-10]}$. Related studies ${ }^{[11]}$ have shown that this type of patient has anatomic abnormalities such as short axis, shallow anterior chamber, narrow frontal angle, hypertrophy of the ciliary body, and relatively large lens. Usually, the eyes are symmetrical. In this study, preoperative examination of these patients found that the anterior chamber was generally shallow; Confirm that abnormal anatomy is the main cause of malignant glaucoma ${ }^{[12]}$.It is suggested that we should be highly alert to the possibility of malignant glaucoma postoperation in patients with such anatomic abnormalities. Since cataract phacoemulsification combined with intraocular lens implantation broke the balance of aqueous humor circulation after surgery, resulting in malignant glaucoma, the mechanism includes the following aspects :(1)The inflammatory reaction after surgery causes the adhesion of the ciliary body to the vitreous body and the reverse flow of the aqueous humor, resulting in the forward migration of the intraocular lens and the iris septum.(2) Surgical trauma causes the bottom of the vitreous base to be separated from the flat part of the ciliary body, causing the aqueous humor to flow back into the vitreous body. (3) The lens capsule and intraocular lens adhere too tightly to form a lens-capsule complex, leaving the vitreous cavity and the posterior chamber without effective liquid flow pathways. The routine surgical treatment of malignant glaucoma including vitreous puncture with 18-20 syringe needle, suction of liquid in the vitreous cavity, injection of liquid or gas into the anterior chamber, YAG laser posterior capsule membrane incision, but the results were often very poor, the success rate is less than $50 \%$.According to the characteristics of the above mechanism, to fundamentally treat malignant glaucoma, the following are needed to do:010Remove the adhesion of the ciliary process to the vitreous body, thereby reducing the posterior chamber pressure 2 2 2 Relieve the posterior capsule membrane and vitreous anterior boundary membrane to obtain a reconstruction of the aqueous humor, flow channel to prevent aqueous humor reflux $\square 3 \square$ Remove the peripheral vitreous body and remove the causes of the anterior iris migration caused by the front of the posterior vitreous body. In this study, 25G vitrectomy was adopted to treat malignant glaucoma, and good results were obtained. Summarizing our experience: (1) Keep a close eye on the condition before surgery. When the treatment with drugs and lasers does not work, early vitrectomy should be performed to prevent anterior angle adhesion and corneal turbidity and loss of surgical opportunities. (2) During the operation, the anterior segment vitreous and the vitreous body around iris incision should be removed to ensure that the flow of aqueous humor is unobstructed and to establish a channel for the flow of water from the vitreous cavity to the anterior chamber. (3) Expand the posterior capsule incision as much as possible to ensure that the anterior chamber can be significantly deepened when the posterior capsule is removed during surgery. Our experience is generally 4-6 mm, avoiding IOL from attaching to the posterior capsule postoperative, and no cases of IOL displacement occur. 
In the past, $20 \mathrm{G}$ vitrectomy had the disadvantages of relatively complicated surgical procedures, more complications, long surgery time, severe inflammation after surgery, and severe discomfort postoperative, resulting in patients and doctors generally avoiding the surgery ${ }^{[13-14]}$. However, with the development of technology, the continuous improvement of the $25 \mathrm{G}$ vitreous cutting system, now vitrectomy incision is smaller(25G about $0.55 \mathrm{~mm}, 20 \mathrm{G}$ about $0.89 \mathrm{~mm}$ ), The surgical procedure is greatly simplified, with fewer complications, rapid healing, no need for sutures, and short surgical time. The total surgical time is $19-45$ minutes, averaging 32 minutes. After the operation, the incision adopts a suture free method. Because of seamless wire stimulation, conjunctiva congestion is not obvious. The patients' comfort is good, and the visual function is restored quickly after surgery ${ }^{[15]}$. The results showed that the BCVA was $0.8 \pm 0.1$, the anterior chamber depth was $2.4 \pm 0.5 \mathrm{~mm}$, IOP was $16.1 \pm 2.5 \mathrm{mmHg}$, and the difference between the above three indicators was statistically significant pre-and postoperation.

To sum up, $25 \mathrm{G}$ minimally invasive vitrectomy can effectively treat malignant glaucoma. It is convenient to operate in surgery. IOP can be significantly reduced. $25 \mathrm{G}$ minimally invasive vitrectomy can effectively improve visual acuity with fewer complications. However, due to the small number of cases in this study, the follow-up time is short, and it is still necessary to further increase the sample size and follow-up time. If necessary, multi-center samples can be collected to evaluate the long-term surgical efficacy.

\section{Conclusions}

Conclusions: Malignant glaucoma after cataract surgery can be treated safely and effectively by $25 \mathrm{G}$ minimally invasive vitrectomy.

\section{Abbreviations}

G gauge BCVA the best corrected visual acuity

IOP intraocular pressure IOL intraocular lens

\section{Declarations}

The authors have no proprietary interest in any of the materials, products, or methods mentioned in this article. The study sponsor had no involvement in the design and conduct of the study; collection, management, analysis, and interpretation of the data; and preparation, review, or approval of the manuscript. 
Ethical Approval and consent to participate

The study was approved by ethics committee of Anhui Medical University and was performed in accordance with the ethical standards laid down in the 1964 Declaration of Helsinki and its later amendments. Written informed consent was obtained from all patients before the procedure.

Consent for publication Not applicable

Availability of data and material The data used to support the findings of this study are included within the article. The datasets used and/or analysed during the current study available from the corresponding author on reasonable request.

Competing interests The authors declare that there is no conflict of interests regarding the publication of this paper.

Funding No funding was obtained for this study

\section{Authors' contributions}

Conceptualization: Shouling Li

Data curation: Fei You

Investigation: Fei You

Methodology: Fei You

Project administration: Shouling Li

Resources: Shouling Li

Software: Fei You

Validation: Shouling Li

Writing - original draft: Fei You

Writing - review and editing: Shouling Li

Acknowledgements Not Applicable 


\section{References}

1. Luntz MH, Rosenblatt M. Malignant glaucoma. Surv Ophthalmol 1987; 32: 73-93.

2. Graefe A. Beiträge zur Pathologie und Therapie des Glaucoms. Albrecht von Graefes Archiv für Ophthalmologie. 1869;15:108-252.

3. Jarade EF, Dirani A, Jabbour E, et al.Spontaneous simultaneous bilateral malignant glaucoma of a patient with no antecedent history of medical or surgical eye disease. Clin Ophthalmol 2014; 8: 1047-1050.

4. Varma DK, Belovay GW, Tam DY, et al. Malignant glaucoma after cataract surgery. J Cataract Refrac Surgery 2014; 40: 1843-1849.

5. Ng WT, Morgan W. Mechanisms and treatment of primary angle closure: a review. Clin Exp Ophthalmol2012; 40: e218-e228.

6. Wang Z, Huang J, Lin J, et al. Quantitative measurement of the ciliary body in eyes with malignant glaucoma after trabeculectomy using ultrasound biomicroscopy. Ophthalmology 2014; 121 : 862869.

7. Zhou C, Qian S, Yao J, et al. Clinical analysis of 50 chinese patients with aqueous misdirection syndrome; a retrospective hospital- based study. J Int Med Res 2012; 40: 1569-1579.

8. Bitrian E, Caprioli J. Pars plana anterior vitrectomy, hyaloido-zonulectomy and iredectomy for aqueous humor misdirection. Am J Ophthalmol 2010; 150: 82-87.

9. Dave P, Senthil S, Rao HL, et al. Treatment outcomes in malignant glaucoma. Ophthalmology. 2013; 120:984-90.

10. Yu J, Chen X, Zhou D, et al. Clinical Efficacy of Ciliary Ring Incision Combined with Modified Partial Pars Plana Vitrectomy for Malignant Glaucoma. Med Sci Monit. 2018;24:3916-3921

11. Madgulal M, Anand N. Long-term follow-up of zonulo-hyaloido-vitrectomy for pseudophakic malignant glaucoma. Indian J Ophthalmol. 2014; 62(12):1115-20.

12. Wu ZH, Wang YH, Liu Y. Management strategies in malignant glaucoma secondary to antiglaucoma surgery. Int J Ophthalmol. 2016; 9(1):63-68.

13. Li M, Wei WB, Li YL, Hui XY, et al. 25-Gauge pars plana vitrectomy for ciliary block(malignant) glaucoma. Int Ophthalmol. 2014;35(4):487-93.

14. Ruben S, Tsai J, Hitchings RA. Malignant glaucoma and its management. Br J Ophthalmol. 1997; 81(2):163-167.

15. Zhou C, Qian S, Yao J, et al. Clinical analysis of 50 chinese patients with aqueous misdirection syndrome: A retrospective hospital-based study. J Int Med Res. 2012; 40:1568-79. 
Page 9/9 